\title{
Les films de propagande sanitaire de Lortac et O'Galop (1918-1919)
}

Health education films by Lortac and O'Galop (1918-1919)

\section{Thierry Lefebvre}

\section{(2) OpenEdition \\ Journals}

Édition électronique

URL : https://journals.openedition.org/1895/3925

DOI : 10.4000/1895.3925

ISBN : 978-2-8218-0984-0

ISSN : $1960-6176$

Éditeur

Association française de recherche sur l'histoire du cinéma (AFRHC)

Édition imprimée

Date de publication : 1 décembre 2009

Pagination : 170-183

ISBN : 978-2-913758-60-5

ISSN : 0769-0959

\section{Référence électronique}


1895 /

$n^{\circ} 59$

décembre

2009

170

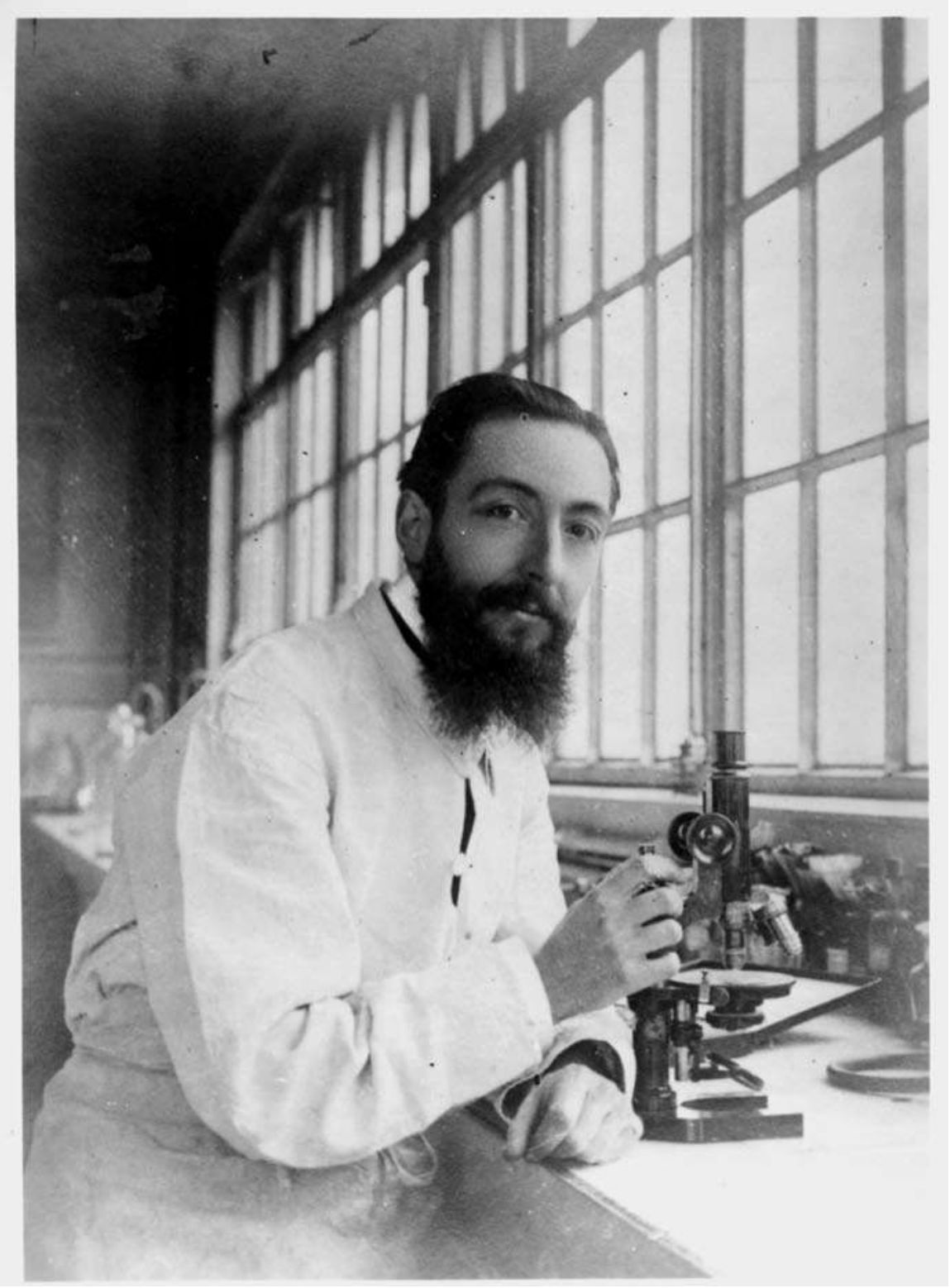

Jean Comandon (1914). 


\title{
Les films de propagande sanitaire de Lortac et O'Galop (1918-1919)
}

\author{
par Thierry Lefebvre
}

De nos jours, les campagnes de santé publique dans les médias audiovisuels sont monnaie courante. Qu'il s'agisse de lutter contre l'alcoolisme, le tabagisme ou le sida, de prévenir les épidémies de grippe ou les infections à papillomavirus, le recours à l'image animée semble désormais incontournable.

Il a fallu pourtant attendre près d'un quart de siècle avant que les pouvoirs publics aient enfin l'idée de convoquer l'outil cinématographique à des fins de propagande sanitaire.

Antérieurement à la Première Guerre mondiale, les rares tentatives n'avaient été le fait que de structures privées, comme, par exemple, les associations antialcooliques ou, à partir de 1914, la Ligue sanitaire française contre la mouche et le rat ${ }^{1}$. Trop faiblement dotées, ces sociétés philanthropiques ne purent malheureusement pérenniser leurs initiatives pionnières. Dans le cadre de cet article, nous nous attacherons à présenter, de manière succincte, le volet cinématographique de la première grande campagne française de santé publique (19171919). Dans un second temps, nous nous intéresserons tout particulièrement à la douzaine de dessins animés qui en constituèrent un des aspects les plus frappants. Ces courts-métrages incisifs, aux publics nombreux et diversifiés, furent conçus par deux animateurs rompus aux techniques publicitaires: O'Galop et Lortac.

\section{Le Comité national d'assistance aux anciens militaires tuberculeux}

L'état de guerre entraîna une prise de conscience tardive des ravages causés par l'endémie tuberculeuse en France. Les troupes furent durablement affectées, puisqu'on estime à 60000 le nombre de soldats réformés pour cette raison durant les deux premières années de guerre.

1 Thierry Lefebvre, "Alcool et ilotes», Sociétés \& Représentations, n 1, novembre 1995, pp. 167-182; Thierry Lefebvre, "Louis-Ferdinand Céline, Raphaël Blanchard et les mouches», dans Thierry Lefebvre (dir. ), Images du réel. La non-fiction en France (1890-1930), 1895, n 18, 1995, pp. 96-105. 
Le 15 avril 1915, le député des Basses-Alpes André Honnorat, préoccupé par ce problème depuis l'avant-guerre, fit voter par la Chambre une résolution relative au traitement des militaires tuberculeux réformés. Six mois plus tard, le 18 octobre, une somme de deux millions de francs fut allouée à cette fin, mais elle se révéla très vite insuffisante. Mises à contribution, les administrations de la Guerre et de l'Intérieur organisèrent des "hôpitaux sanitaires " réservés aux tuberculeux, mais aussi des «stations sanitaires » dont l'objet était "d'assurer aux «blessés de la tuberculose » non point un traitement curatif complet, mais surtout l'éducation antituberculeuse de manière à ce que le malade ne soit plus exposé à contaminer son entourage et les milieux ambiants ${ }^{2}$.

C'est à partir de 1916 que furent édités les premiers tracts et affiches formulant des « conseils d'hygiène » simples, à l'attention des patients atteints d'affections des voies respiratoires: "Évitez les poussières", "Ne crachez pas par terre", "Fuyez les deux poisons: l'alcool et le tabac», "Lavez-vous tous les matins», "Brossez bien vos dents», etc. Ces préceptes simples, à l'époque insuffisamment diffusés au sein de la population française, servirent de matrice à la campagne cinématographique dont il va être question.

Le 26 mai 1916 fut constitué le Comité départemental d'assistance aux anciens militaires tuberculeux du département de la Seine. Le sénateur Auguste Ranson en assumait la présidence, tandis que le $\mathrm{D}^{r}$ Léon Bernard et Léonie Chaptal avaient en charge le secrétariat. À l'instar des autres comités départementaux, cette association privée était chapeautée par un Comité national auquel revenait la tâche de coordonner l'ensemble des structures régionales, d'organiser la collecte d'argent et de concevoir la propagande d'hygiène.

Placée sous le haut patronage du président de la République et présidée par Léon Bourgeois, cette association philanthropique, reconnue d'utilité publique le 14 septembre 1916, organisa le 4 février 1917 la première «Journée nationale des tuberculeux anciens militaires» dont le slogan était: «Sauvons-les». La collecte d'argent fut satisfaisante et stimula les initiatives, si bien que le 11 mars, le $\mathrm{Dr}$ Jean Comandon, qui servait depuis le début de la guerre sous les drapeaux, fut mis à la disposition du Comité national " pour la préparation de films cinématographiques destinés à la propagande ". À vrai dire, l'idée d'une campagne coordonnée était en germe depuis quelques mois, en particulier grâce aux efforts déployés par le professeur Raphaël Blanchard, président de la Ligue sanitaire française contre la mouche et le rat, et grand adepte du cinéma éducateur. Trois films commandités par cette association, réalisés par Pathé et Gaumont, avaient été présentés au ministère de I'Intérieur dès le 27 janvier 1917, et il semble bien que Comandon soit intervenu, au cours 2 «Sur I'hospitalisation des militaires tuberculeux", Revue d'hygiène et de police sanitaire, $\mathrm{n}^{\circ} 40,1918$, p. 235. 


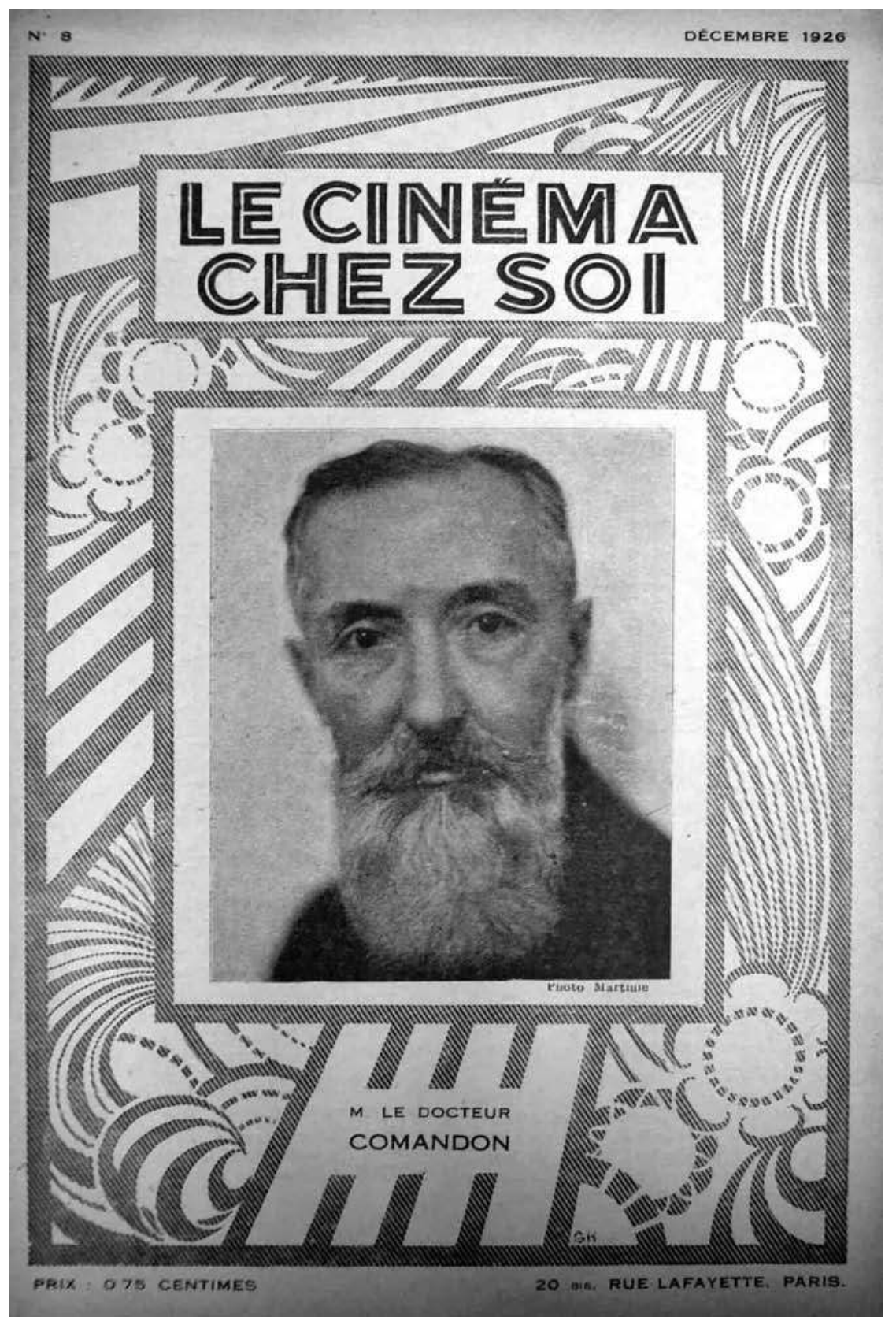

Jean Comandon, le Cinéma chez soi, $\mathrm{n}^{\circ} 8$, décembre 1926. 


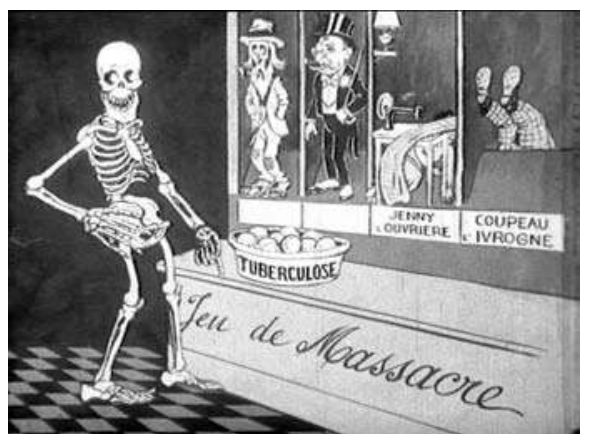

1. Lortac, la Tuberculose menace tout le monde, 1918.

$1895 /$

$\mathrm{n}^{\circ} 59$

décembre

2009

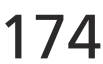

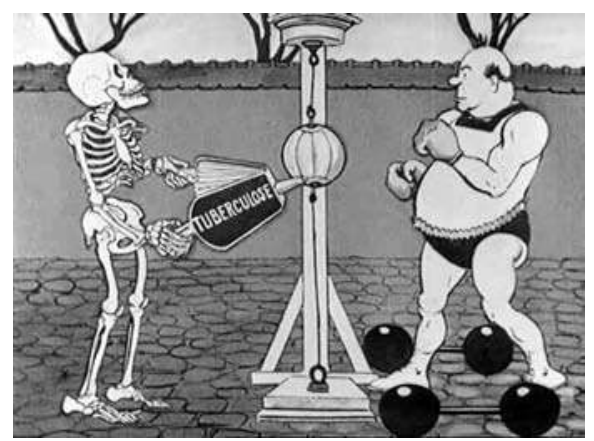

2. Lortac, Pour résister à la tuberculose, soyons fort, 1918. de quelques-unes de ses permissions, dans la mise en forme définitive de l'un d'entre eux, la Mouche domestique ${ }^{3}$.

En pratique, muni de son ordre de mission, le médecin-cinéaste retourna dans le laboratoire des studios Pathé à Vincennes: il en réquisitionna le matériel technique et, flanqué de son nouvel opérateur Georges Régnier, sillonna la France pour y tourner une première série d'une douzaine de moyens-métrages. Furent ainsi visités et documentés par le cinéma: le dispensaire Léon-Bourgeois, les sanatoriums de Bligny et Roscoff, la station sanitaire de Pignelin, l'école de plein air du PlessisRobinson, la pouponnière de Remardray, etc. Ce premier ensemble de films, dont la finalité était de témoigner de l'effort fourni par le Comité national et ses antennes départementales en vue de doter la France d'un véritable "arsenal» antituberculeux, fut mis à la disposition des autorités sanitaires, mais aussi de la Commission américaine de prévention contre la tuberculose (mission Rockefeller) ${ }^{4}$, dès le début de l'année 1918.

Parallèlement, Jean Comandon mit en œuvre une dizaine de saynètes visant à inculquer quelques notions élémentaires d'hygiène: Ne crachez pas par terre, Lavez-vous les mains avant le repas, Ne mouillez pas vos doigts pour tourner les pages, etc. Ces films très courts, généralement tournés en intérieurs avec des acteurs apparemment professionnels, consistaient en la mise en image, dépouillée de toute afféterie, de préceptes déjà diffusés sous forme de tracts et de circulaires, comme nous l'avons vu'5.

3 Thierry Lefebvre, Cinéma et discours hygiéniste (1890-1930), Th. Doctorat Cinéma et Audiovisuel, Université Paris 3, 1996, p. 77.

4 « Commission for the Prevention of Tuberculosis in France », émanation de l'International Health Board de la Rockefeller Foundation.

5 Signalons également quelques autres films produits par Comandon durant cette période: puériculture (deux titres), propagande contre les animaux nuisibles (trois films), ainsi que l'adaptation d'un film d'hygiène américain: Une dent propre ne se carie jamais. 


\section{Une série de dessins animés hygiénistes}

Le troisième pan de la production hygiéniste coordonnée par Jean Comandon se composait de dix dessins animés qui furent répartis comme suit:

- Un premier sous-groupe de quatre films spécifiquement " antituberculeux » : le Taudis doit être vaincu (rebaptisé après la guerre l'Aigle boche est vaincu, la tuberculose doit l'être aussi ); Pour résister à la tuberculose, soyons forts; la Tuberculose menace tout le monde et Comment on attrape les maladies contagieuses.

- Un deuxième sous-ensemble de quatre films de "propagande antialcoolique »: le Circuit de I'alcool; Petite cause, grands effets; la Tuberculose se prend sur le zinc et l'Oubli par l'alcool (Doit \& avoir).

- Enfin, deux courts-métrages «isolés »: I'un de "propagande antivénérienne » (On doit le dire), l'autre de "propagande contre les animaux transmetteurs de maladies» (la Mouche).

Tous ces films furent dûment répertoriés dans le catalogue des Films médicaux et scientifiques de Pathé Consortium Cinéma - Séries Gaston Doin $n^{\circ} 1$, édité vers 1920, ainsi que dans sa réédition complétée, revue et corrigé de 1924 (Liste des films médicaux et scientifiques de Pathé Consortium Cinéma, pris sous la direction du Dr Comandon - Séries Gaston Doin $n^{\circ} 2$ ).

S'ils constituent à eux dix un corpus cohérent, dans lequel la mission Rockefeller puisa d'abondance, il n'en demeure pas moins que ces films paraissent avoir été réalisés à des périodes et dans des contextes légèrement différents.

Si l'on s'en tient à la numérotation adoptée par le second catalogue Doin, les quatre premières bandes éditées (dites de "propagande antituberculeuse ») le furent indiscutablement sous l'égide du Comité national d'assistance aux anciens militaires tuberculeux, possiblement durant les premiers mois de l'année 1918. Se pose néanmoins, pour ces premiers dessins animés, un délicat problème d'attribution: trois de ces courts-métrages ( /a Tuberculose menace tout le monde, le Taudis doit être vaincu et Pour résister à la tuberculose, soyons forts) présentent en effet le même personnage récurrent, un grand squelette muni d'un soufflet symbolisant la tuberculose. Un carton-titre, datant apparemment de la fin des années 1910 ou du tout début des années 1920, attribue la Tuberculose menace tout le monde à Lortac. En revanche, un titre surajouté (il y a probablement plus d'une trentaine d'années) à la seule copie alors en circulation du Taudis doit être vaincu, attribue ce film à O'Galop, qui plus est avec une date erronée (1912). À moins d'envisager une collaboration étroite entre $\mathrm{O}^{\prime}$ Galop et Lortac pour cette série (ce qui n'est pas à exclure), nous nous trouvons visiblement devant une contradiction flagrante. Aussi, dans l'état actuel de 
$1895 /$

$n^{\circ} 59$

décembre

\section{Le recours à l'animation}

L'arrivée en France de la Commission américaine de préservation contre la tuberculose en août 1917, la création par cette dernière d'un «bureau de propagande et de publicité » dont la direction incomba au Dr Selskar M. Gunn, professeur d'hygiène à l'Université de Boston, puis la mise en œuvre, à partir de mars 1918, d'un vaste programme itinérant d'«éducation populaire » axé sur les projections cinématographiques, firent évoluer de façon sensible les objectifs que s'était assigné, du moins à l'origine, le Comité national d'assistance aux anciens militaires tuberculeux. En effet, comme l'indiquait son intitulé, cette association s'était d'abord constituée afin de venir en aide aux soldats «blessés de la tuberculose». La production de films, telle que I'avait conçue au départ Jean Comandon, visait donc trois objectifs principaux: éveiller l'empathie des futurs donateurs; témoigner de la tâche accomplie en documentant les premières réalisations d'envergure du Comité (sanatoriums, stations sanitaires, écoles de plein air, etc.); et inculquer aux réformés militaires des mesures simples d'hygiène susceptibles d'être relayées ensuite auprès de leurs famille et entourage.

6 Notre propos ne visant absolument pas à démêler les intentions auctoriales des uns et des autres, mais plutôt à exposer les conditions de production de ces films et leurs usages, la question est - il est vrai secondaire.

$7 \mathrm{Cf}$. note 1 (référence 2). Signalons aussi la réalisation d'un autre film, celui-là en images réelles: Faisons la guerre aux rats.

8 Henri Gougerot, futur président de la Société française de prophylaxie sanitaire et morale, qualifiera en 1928 ce film de «remarquable». 


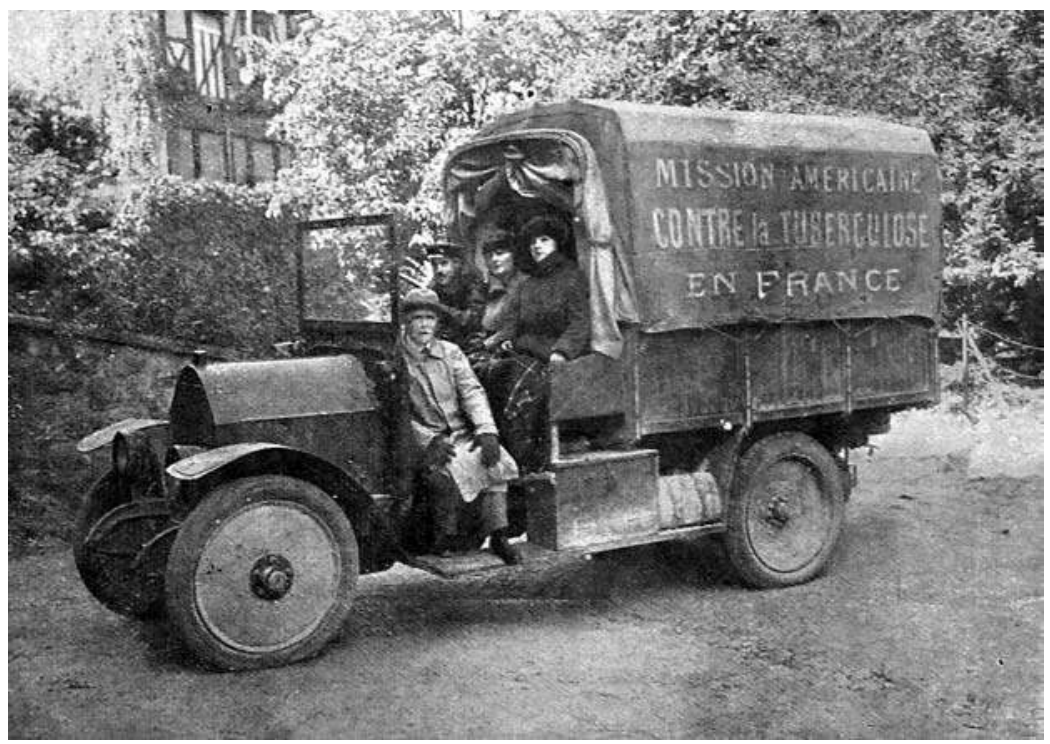

$1895 /$ $n^{\circ} 59$ décembre 2009

Camion de la mission Rockefeller, Cinémagazine, 23 décembre 1921.

La Commission américaine, imitant en cela les expériences menées aux États-Unis depuis le début des années 1910, décida d'élargir le ciblage de sa propagande. Furent désormais visés les notables, les commerçants, les ouvriers, les militaires, les femmes, mais aussi et surtout les enfants scolarisés, considérés comme prioritaires dans la mesure où on les pensait capables de prescrire de nouveaux comportements d'hygiène au sein de leurs familles. Comme le rappellera plus tard Alexandre Bruno, membre du comité exécutif de la Mission Rockefeller, «la propagande débutait par des causeries dans les écoles. Une causerie aux enfants de 15 à 20 minutes était suivie d'une démonstration cinématographique spécialement adaptée, gaie, comique souvent, toujours instructive. Quelle joie, quel enthousiasme, quelles figures épanouies parmi ce petit monde ! $» 9$.

Dans un article paru en 1921 dans Cinémagazine, Robert Marcel-Desprez vantait les mérites du «cinéma que tout le monde comprend, qui intéresse même les illettrés, et marque d'une trace indélébile le souvenir [...]». Ainsi, constatait-il, " on siffle un orateur, on ne conspue pas un film, on le discute $[\ldots] » 10$.

En novembre 1918, un journaliste anonyme du Matin témoigna d'une séance de projection

9 Alexandre Bruno, Le Rôle de la mission Rockefeller dans l'organisation antituberculeuse en France, 1917 à 1923. Th. Doct. Médecine, Paris, 1925.

10 Robert Marcel-Desprez, «La propagande par le cinéma », Cinémagazine, 23 décembre 1921, p. 16. 
de la Commission américaine de préservation contre la tuberculose, en première page du quotidien parisien. Son témoignage était éclairant:

[...] Et le cinéma commence. Il y a de tout dans le spectacle: des films d'art et des films enfantins, tel celui qui montre un buveur attablé devant sa bouteille favorite, la mort qui entre, lève le bras, lance un projectile contre le buveur qui tombe à la renverse, mort. Changement de vue. Nous voici dans un champ où un solide paysan laboure. La mort survient. À lui aussi, elle jette un projectile meurtrier. Mais le laboureur porte en soi la santé comme une cuirasse et la balle rebondit. Elle tombe à terre: la mort est vaincue. Vous riez? Tant mieux. Ils riaient aussi les spectateurs des grandes conférences et des quarante petites causeries que, dans une seule ville, comme

Même si son compte-rendu du film projeté ce jour-là laissait à désirer (on reconnaît malgré tout le principe général de la Tuberculose menace tout le monde), le journaliste avait bien compris ce qui faisait l'efficacité redoutable de ces images: à savoir, leur capacité à marquer au plus profond les esprits. À l'instar des réclames pour les poudres de Cock qui, depuis une dizaine d'années, intimaient au public l'ordre de "s'enfoncer bien ceci dans la tête", I'imagerie des films diffusés par la Mission Rockefeller se voulait volontiers «mécaniste».

On se rappelle que les pavés publicitaires pour les poudres de Cock donnaient à voir le visage d'un gros homme à la mine enjouée: une main vigoureuse, armée d'un lourd marteau, lui enfonçait un large coin dans le crâne, - illustration on ne peut plus claire de ce que l'on désignera plus tard par l'expression «matraquage publicitaire » ${ }^{12}$. Semblablement, dans les

11 «Science et réclame. La tuberculose soignée par la publicité. La médecine propage par le spectacle les conquêtes du laboratoire ", le Matin, 30 novembre 1918, p. 1.

12 Sur I'histoire de cette campagne publicitaire emblématique conçue apparemment par Julien Charles Pinat (dit Jep), lire: Thierry Lefebvre, «ll y a cent ans: I'homme au marteau», Revue d'histoire de la pharmacie, $\mathrm{n}^{\circ} 350,2^{\mathrm{e}}$ trimestre 2006, pp. 199-204. Rappelons-en le slogan impérissable: «Maladies d'estomac. Enfoncez-vous bien ceci dans la tête: les poudres de Cock sont souveraines dans toutes les affections de I'estomac, du foie, de l'intestin. » 
films de la Commission américaine, la Mort, « incarnée » par un grand squelette sarcastique, aspergeait de bacilles tuberculeux ses victimes, tandis qu'un médecin pointait vers elle un «canon d'hygiène», allégorie de l'«arsenal thérapeutique» que la Nation était en train de constituer (le Taudis doit être vaincu). Ailleurs, I'endémie tuberculeuse était assimilée à un véritable «jeu de massacre» (la Tuberculose menace tout le monde); seule la "vie saine" parvenait à y mettre un terme, en la mettant littéralement "knock-out» (Pour résister à la tuberculose, soyons forts).

\section{O'Galop et Lortac}

Ces images avaient été conçues par deux dessinateurs visiblement très au fait des techniques et exigences publicitaires. O'Galop, en particulier, avait imaginé le personnage emblématique du bibendum Michelin dès 1898, avant de vanter tour à

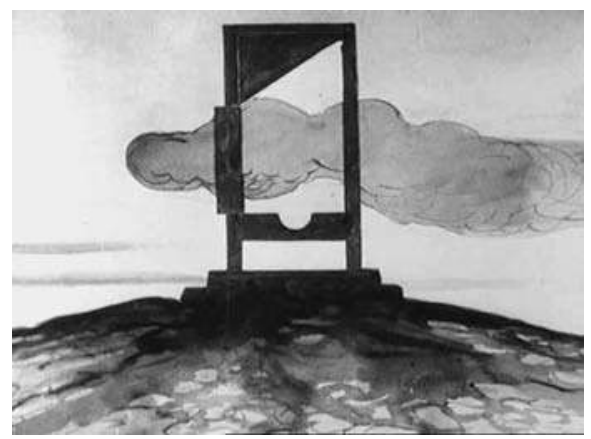

1. O'Galop, Petite causes, grands effets, 1919.

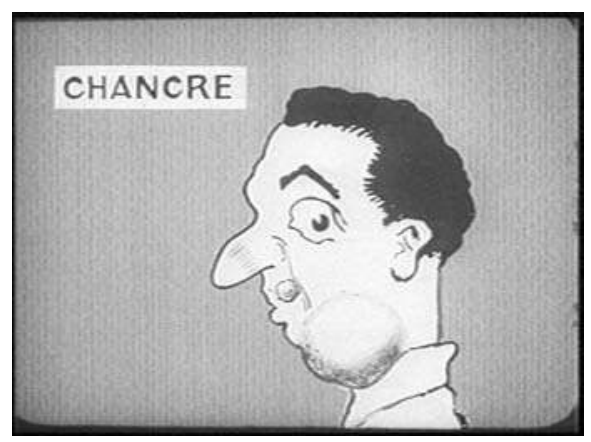

2. O'Galop, On doit le dire, 1918. tour les mérites de l'Eau de Suez, de la pile Hydra, des bougies Macquaire, de l'Akesol Muzi, de la pâte dentifrice Gibbs, etc., dans la presse comme par l'affiche.

Lui et Lortac menèrent donc en parallèle, chez Pathé, une activité de propagandiste au service de la santé publique et une production à visée plus distractive (et probablement plus lucrative): séries Mécanicas ou Toto pour Lortac; séries Touchatout et bientôt Bécassotte pour O'Galop.

Leurs petits films éducatifs présentaient toutes les vertus d'une bande publicitaire: ils étaient courts, animés, plutôt humoristiques, montraient le "produit» en action (en l'occurrence, les bons comportements d'hygiène, toujours opposés aux mauvais) et ne lésinaient pas sur les images-choc: squelette, "canon à hygiène ", tête de mort, guillotine, asile, présentations d'individus présentés comme "tarés», criminels ou épileptiques, etc., tout cela à des fins d'édification.

La technique du «before-after » était également invoquée: le logis sale et poussiéreux se transformait en appartement modèle ensoleillé dans le Taudis doit être vaincu, le syphilitique d'On doit le dire recouvrait la santé après la prescription d'un traitement adapté; plus 


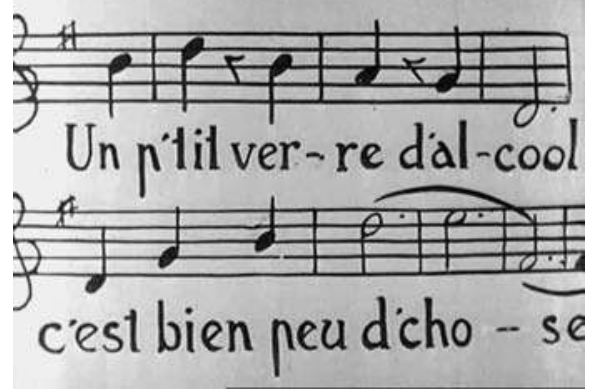

1. O'Galop, Petite causes, grands effets, 1919.

$1895 /$

$\mathrm{n}^{\circ} 59$

décembre

2009

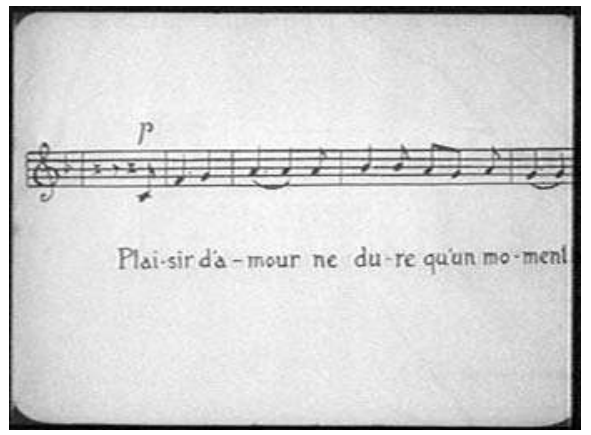

2. O'Galop, On doit le dire, 1918.

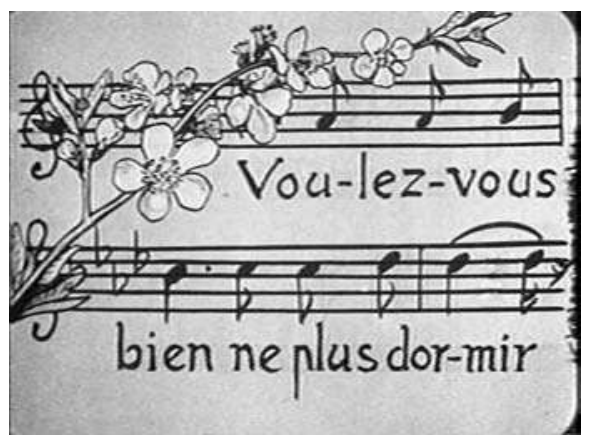

3. O'Galop, la Mouche, 1919. subtil, I'alcoolique de l'Oubli par l'alcool se réveillait au terme d'un mauvais rêve et décidait de se réformer sur le champ et de renoncer à l'alcool.

Certains films témoignaient aussi d'une volonté indéniable d'interactivité, et donc d'une prise en compte de leurs futures conditions de diffusion dès la rédaction du scénario. On doit le dire insérait ainsi dans sa continuité les premières notes de Plaisir d'amour. La Mouche s'ouvrait sur le début de la partition de Quand l'oiseau chante. L'accompagnement musical était ainsi dicté par les images, et la reprise en chœur par l'assistance des paroles de ces chansons était encouragée. Petite cause, grands effets débutait par une chanson à boire («Un p'tit verre d'alcool, c'est bien peu de chose... "). Toujours dans la Mouche, l'allusion finale à une célèbre fable de la Fontaine - l'Ours et l'Amateur de jardins - visait certes à amuser les enfants, mais aussi, et surtout, à les renvoyer à un grand classique de la littérature inscrit au programme de leurs études...

\section{Le rôle de Jean Comandon}

Quel fut précisément le rôle de Jean Comandon dans cette production si particulière?

Ces dessins animés, nous l'avons vu, figurèrent dans la Liste des films médicaux et scientifiques de Pathé Consortium Cinéma (séries Gaston Doin $n^{\circ} 2$ ), sous l'intitulé «bandes de propagande d'hygiène sociale ». Il y était expressément précisé, en couverture: «pris sous la direction du Dr Comandon ».

On peut néanmoins penser que le rôle du médecin se borna à formuler un certain nombre de prescriptions et de bonnes pratiques édictées par le Comité national d'assistance aux anciens militaires tuberculeux, puis par la Commission américaine de préservation contre la tuberculose et la Croix-Rouge américaine. Un exemplaire du premier catalogue Doin, 
visiblement annoté par le cinéaste et conservé aux archives de l'Institut Pasteur, stipule que ces films antituberculeux furent réalisés sous la responsabilité du Pr Maurice Letulle, du Dr Léon Bernard et du Pr Selskar Gunn, et que la Mouche fut conçue sous le patronage du Pr Raphaël Blanchard. Jean Comandon se portait donc garant de la concordance des bandes produites sous sa direction avec les préceptes et informations validés par les organismes commanditaires. À ce titre, on peut légitimement le considérer comme le coscénariste de I'ensemble de ces films.

Deux de ces dessins animés font appel à des images documentaires tournées par Comandon. Il s'agit de la Mouche et d'On doit le dire, dont nous avons signalé le caractère un peu plus tardif. Nous avons montré, par ailleurs, que les prises de vues microcinématographiques de spirochète pâle insérées dans On doit le dire provenaient du film princeps du cinéaste, Spirochaeta

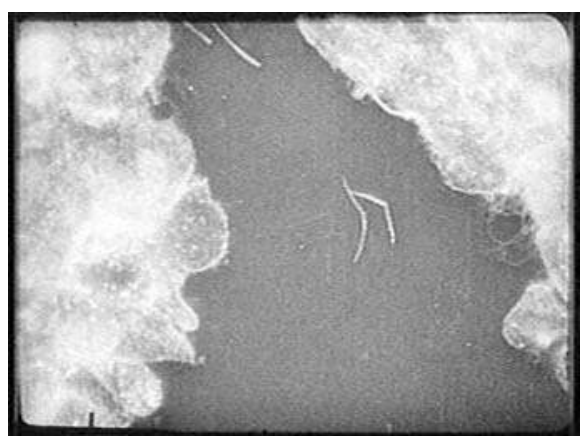

1. O'Galop, On doit le dire, 1918.

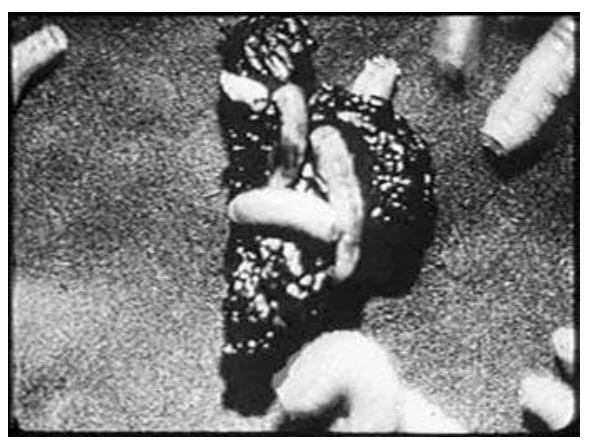

2. O'Galop, la Mouche, 1919.

pallida tourné en 190913. Pour ces raisons et uniquement pour ces deux films, Jean Comandon doit être crédité en tant que coréalisateur. Coscénariste des huit autres, il en supervisa la mise en forme définitive et en signa le «bon à développer».

\section{La réception des films}

Ces films eurent sans conteste un impact considérable sur le jeune public. Dans un article daté de 1924, Lucien Viborel se voulait résolument optimiste à leur égard et imaginait un enfant convertissant son père au retour d'une projection scolaire: «Le Maître nous a montré, au cinéma, des microbes. Le microbe de la tuberculose est comme un bâtonnet. Je l'ai bien vu; il existe, et il est même très mauvais... » ${ }^{14}$.

La réalité devait certes être moins idyllique. En 1925, Louis Gablin, instituteur adjoint à l'École

13 Thierry Lefebvre, «Le cinéma contre la syphilis. Pré-enquête sur une lignée cinématographique », dans Emmanuelle Toulet, Christian Belaygue (dir.), CinéMémoire. 3e Festival international. Films retrouvés - Films restaurés, Paris, CinéMémoire, 1993, pp. 160-174.

14 Lucien Viborel, «Le rôle de l'enfant dans la croisade sanitaire », la Vie saine, n 7, février 1924, p. 3. 
d'application de Saint-Lô, eut par exemple l'idée de projeter certains de ces films devant une assemblée d'enfants âgés de sept à onze ans. À la fin de la présentation, il les interrogea sur ce qu'ils avaient vu:

"Qu'est-ce que la tuberculose?», demanda-t-il. "C'est une maladie, répondirent les enfants. On la gagne. On peut l'attraper. Ça vous entre par la bouche.»

Deuxième question: "À quoi est due cette maladie? " Réponses: "C'est des petites bêtes qu'on avale. Ça va dans les poumons. Ce sont des microbes. » L'élève Maca (onze ans) précise: «Non, ce sont des germes, de petits champignons... » Le Maître demande: "Comment se manifeste la tuberculose?" Réponses: "On est maigre. On tousse. On a de grands yeux. On a de la fièvre. Ça fait cracher. Ça fait des soupapes dans les poumons, etc., etc. » [On reconnaît les symptômes évoqués dans Pour vaincre la tuberculose, soyons forts.]

Louis Forest commentait cette expérience: «Certains films ont été bien compris; mais d'autres réponses révèlent aux propagandistes par le cinéma qu'il ne convient pas d'être trop subtil... ». L'usage de la métaphore, si courant dans ces petits dessins animés, pouvait en effet être source de quiproquos. "Un élève répond: «Quand il y a la tuberculose, il faut prendre un canon! ». La scène représentée par le film [le Taudis doit être vaincu] montrait un canon symbolique sur lequel était écrit le mot «hygiène». Le petit n'avait vu que le canon. Il s'était dit que l'hygiène n'avait aucun rapport avec l'artillerie... ".

Autre exemple: dans la Tuberculose menace tout le monde, un athlète terrassait le squelette symbolisant la maladie. "La petite Godreuil conclut que pour guérir, il faut se battre... ${ }^{15}$ ». II n'empêche que ces dessins animés marquèrent profondément une génération de petits provinciaux qui, pour un grand nombre d'entre eux, découvrirent le cinéma au travers des aventures héroïques, et somme toute amusantes, d'ilotes et de personnages voués au culte de l'Hygiène. Les conséquences de ce "premier contact» avec le média cinématographique furent sans doute importantes, même si elles s'avèrent difficiles à évaluer.

Dans la seconde moitié des années 1920, seuls deux de ces films furent réédités au format 9,5 mm pour le projecteur Pathé-Baby: la Mouche (rebaptisé Guerre aux mouches!, n 1182 du catalogue de 1931) et la Tuberculose se prend sur le zinc ( $\left.n^{\circ} 1190\right)$. On ne connaît pas les motifs qui guidèrent cette sélection quelque peu drastique. Notons cependant que ces deux bandes avaient pour même auteur O'Galop, et que ce dernier était explicitement crédité dans la notice de Guerre aux mouches! («dessins animés d'O'Galop», était-il précisé en gras dans le catalogue Pathé-Baby de 1931).

15 Louis Forest, «La critique des gosses», la Vie saine, $n^{\circ} 29$, décembre 1925, p. 7. Le texte est repris dans Lucien Viborel, la Technique moderne de propagande d'hygiène sociale, Paris, Éditions de la Vie saine, 1930, p. 650. 


\section{Conclusion}

Depuis une quinzaine d'années, ces petits films, désormais retrouvés, identifiés et en cours de restauration aux Archives françaises du film du CNC, ont été régulièrement projetés devant des publics variés. Nous eûmes ainsi le plaisir de les présenter et de les commenter à de nombreuses reprises: par exemple, à la Cinémathèque française dans le cadre du festival CinéMémoire de 1993, au Forum des Images en 1999, à I'occasion du cycle "Petite histoire de I'hygiène au cinéma » à la Cité des Sciences en 2004, en ouverture du XVIe Festival de la communication médicale de Deauville en 2005, dans l'amphithéâtre du musée Curie en 2006, ou encore, plus récemment, à l'occasion des Rendez-vous de I'Histoire de Blois de 2009. Productions amusantes (et parfois si inquiétantes!) d'une époque aujourd'hui révolue, ces dessins animés témoignent des croyances et pratiques sanitaires qu'il s'agissait alors d'insuffler dans la population française. Très diserts sur la question des «maladies sociales » (alcoolisme, tuberculose et syphilis), ils n'en passèrent pas moins sous silence la tragédie sanitaire qui résulta de la pandémie de grippe «espagnole» de 1918-1919. 